\title{
Nelson Goodman in der Diskussion
}

\author{
Von SABINE AMMON (Berlin)
}

JAKOB STEINBRENNER / OLIVER R. SCHOLZ / GERHARD ERNST(HG): SYMBOLE, SYSTEME, WELTEN - STUDIEN ZUR PHILOSOPHIE NELSON GOODMANS. Synchron. Wissenschaftsverlag der Autoren, Heidelberg 2005, 280 S.

Mit dem vorliegenden Sammelband haben es sich die Herausgeber zur Aufgabe gemacht, das Gesamtwerk eines der großen Denker des 20. Jahrhunderts zu würdigen. Nelson Goodman (1906-1998), der zu Unrecht noch immer im Schatten der großen Namen im Umfeld der analytischen Philosophie steht, hat in vielen philosophischen Bereichen wegweisende Werke hinterlassen. Aufsehen erregte The Structure of Appearance (1951) als Beitrag zur nominalistischen Logik, weithin bekannt wurde Goodman mit seinem Buch Tatsache, Fiktion und Voraussage (1954) zum Induktionsproblem. Sprachen der Kunst (1968) avancierte innerhalb der Ästhetik zum Klassiker, in Weisen der Welterzeugung (1978) und Revisionen: Philosophie und andere Künste und Wissenschaften (1988, zusammen mit Catherine Elgin) wird ein provokanter Ansatz zur Erkenntnistheorie und Metaphysik erarbeitet - um nur die wichtigsten Arbeiten zu nennen.

Der Sammelband greift die großen Themenstränge aus Goodmans Euvre auf und stellt sie in neue Zusammenhänge. Den Auftakt bildet ein einleitender Beitrag von Oliver Scholz, der sorgfältig recherchiert über Leben, Werk und Rezeptionsgeschichte von Nelson Goodman berichtet. Ergänzt wird die Vorstellung durch einen Beitrag von Goodman selbst zur Wirkung von Kunstwerken und einen Aufsatz seiner engsten Mitarbeiterin Catherine Elgin. Diese beiden Beiträge liegen durch die Übersetzung von Gerhard Ernst nun erstmals auf Deutsch vor. Goodmans Nominalismus für die Behandlung der Mathematik ist Thema von Karl-Georg Niebergall, Gerhard Ernst schließt seine Überlegungen an die Induktionsproblematik an, Donatus Thürnau baut Gedanken der Sprachen der Kunst aus, Matthias Haase diskutiert Aspekte des Spätwerks. Jakob Steinbrenner untersucht die Exemplifikation, ebenso wie Dieter Mersch, der einen Vergleich mit Wittgenstein anstellt. Einen Schwerpunkt bilden die Beiträge zur Ästhetik, und hier insbesondere der Bildtheorie, mit Aufsätzen von Andrea Esser, Thomas Hölscher, Jasper Liptow, Klaus Sachs-Hombach und Jens Kulenkampff. Abgerundet wird der Band mit dem Abdruck eines der seltenen Interviews mit Goodman, welches Karlheinz Lüdeking 1990 führte und das für den Sammelband neu überarbeitet wurde.

Die Reichhaltigkeit der Philosophie Goodmans ebenso wie das große Potenzial seiner Gedanken wird an diesen Beiträgen sichtbar - ein Schatz, der noch lange nicht vollständig gehoben ist. Gemeinsam ist den sehr unterschiedlichen Aufsätzen die frische und unbekümmerte Herangehensweise, die weit entfernt ist von jeder Verklärung. Vergleiche werden gezogen, kritisch Positionen abgeklopft und Grenzen ausgelotet, die Anschlussfähigkeit wird geprüft: ein Vorgehen, das im Folgenden exemplarisch an drei Beiträgen gezeigt werden soll.

Donatus Thürnau untersucht in seinem Beitrag Die Sprache des Körpers - Nelson Goodmans Analyse mimisch-gestischer Zeichen das Potenzial des symboltheoretischen Ansatzes für die aktuelle Debatte der Körpersprache. Da Goodman selbst keine eigenständige Untersuchung zu diesem Bereich vorgenommen hat, muss sich der Autor für die Rekonstruktion 
auf wenige Textpassagen stützen. So kann Thürnau Grundformen symbolischer Funktionsweisen wie Denotation und Exemplifikation im Bereich „körpersprachlicher Phänomene“ aufzeigen, Ausdruck als wichtige Variante der Exemplifikation findet er im Sichtbarmachen von Gefühlen.

Aufbauend auf diesen Befund, kontrastiert der Autor den symboltheoretischen Ansatz mit der Ausdruckslehre, die Anfang des 20. Jahrhunderts ihre Blütezeit erlebte. Körperlicher Ausdruck wird hier in der Regel als Symptom gedeutet. Doch unbestreitbar trägt nach Thürnau der Ausdruck von Freude, Trauer oder Ärger auch konventionelle Züge. Er folgert daraus, dass Ausdruck anders als durch kausale Verursachung bestimmt werden muss - ein Weg, der den Anhängern der Ausdruckslehre zwangsläufig verschlossen bleibt.

Abschließend prüft Thürnau die Vorzüge der Theorie Goodmans in Bezug auf die heutige populärwissenschaftliche Diskussion, die er in großen Teilen der Ausdruckslehre verhaftet sieht. Körpersprache als Ausdruck von Gefühlen und Mitteilungen an den Empfänger werden biologisch determiniert und damit universell gültig gedeutet. Thürnau verortet Goodman dagegen bei den Kulturrelativisten, die eine kulturelle Abhängigkeit körpersprachlicher Elemente betonen. Nur dort, wo körperliche Phänomene als Symbole erfasst werden, können wir von einer "Körpersprache" im engeren Sinn reden.

Die Ausführungen Thürnaus zeigen, dass mit dem Thema mimisch-gestischer Zeichen im Rahmen der Symboltheorie eine interessante Spur gelegt ist, die sich lohnt weiterzuverfolgen. Sehr aufschlussreich kann es werden, wenn die Rolle des Körpers auch im Zusammenhang mit der Herausbildung neuer Zeichenrelationen betrachtet wird. Sind Symbolsysteme kaum entwickelt, kommt exemplifizierenden Vorgängen eine entscheidende Bedeutung zu, wie Goodman am modernen Tanz zeigt. Körperbewegungen haben hier einen entscheidenden Anteil am Individuierungsprozess - ein Befund, der sich möglicherweise auch auf andere Bereiche übertragen lässt.

Jens Kulenkampff hinterfragt in seinem Beitrag Sind Bilder Zeichen? den in der Bildwissenschaft weithin akzeptierten Ansatz Goodmans, der bildliche Darstellung auf die Denotation zurückführt. Da Bilder vielfältige Funktionen übernehmen können, ist für den Autor entscheidend, was etwas zu einer bildlichen Darstellung macht.

Um diese Frage innerhalb der Symboltheorie zu beantworten, muss sie nach Kulenkampff zwei Voraussetzungen klären. Erstens ist sicherzustellen, dass es sich um ein Symbol handelt. Da Goodman seine Theorie bildlicher Symbole analog zu sprachlichen Prädikaten entwickelt, muss er eine vergleichbare Funktionsweise der Bilder nachweisen - was ihm laut Kulenkampff auch gelingt. Unsere Rede von bildlicher Darstellung wird allerdings da zweideutig, wo der Sachbezug leer ist. Der Autor sieht in der Lösung Goodmans zwar eine gelungene Argumentation, um fragwürdigen ontologischen Annahmen aus dem Weg zu gehen, aber keine Antwort auf die Ausgangsfrage.

Zweitens müssen die Unterschiede zwischen Bildern und Prädikaten deutlich gemacht werden, die jeweils unterschiedlichen Systemen angehören. Für Goodman liegen die Kriterien der Differenzierung im System begründet, die Besonderheit bildlicher Systeme zeigt sich in einem „Mangel an syntaktischer Artikulation“, der Dichte. Hieraus folgert Kulenkampff, dass darstellende Bilder keine Syntax besitzen, der Abgrenzungsversuch läuft demnach ins Leere.

Das Fazit Kulenkampffs fällt deutlich aus: Goodmans Ansatz versagt bei der Frage, was eine bildliche Darstellung ist. Da diese Frage jedoch jeder Bildertheorie vorausgehen muss, kann die Symboltheorie nicht genügen. Eine Lösung für dieses Problem hat der Autor nicht, 
sieht aber einen möglichen Weg in der Berücksichtigung des Herstellungsvorganges von Kunst.

Sicherlich greift hier die Kritik an Goodman zu kurz. Fälschlich wird die Dichte eines Systems und die damit verbundene Unschärfe mit einem Nichtvorhandensein von Syntax gleichgesetzt, die Überlegungen zum Gesamtsystem werden allein auf syntaktische reduziert. Dennoch verweist Kulenkampff mit seiner Kritik auf einen wichtigen Punkt: Es ist die Frage, wie weit das Instrumentarium aus syntaktischen und semantischen Merkmalen, das uns Goodman zur Analyse der unterschiedlichen Symbolsysteme an die Hand gibt, überhaupt trägt. Möglicherweise sind differenziertere Werkzeuge notwendig, um Unterschiede zwischen so unterschiedlichen Bereichen wie Sprache und bildlichen Systemen befriedigend zu benennen, oder Gemeinsamkeiten im Bereich bildlicher Darstellung aufzufangen, die sich im groBen Spektrum zwischen Piktogrammen und künstlerischer Avantgarde abspielen.

Trotz der scheinbaren Heterogenität und der Themenvielfalt in Goodmans Werk gibt es Gedankenfiguren, die sich durch alle Bereiche ziehen. Gerhard Ernst hat sich in seinem Beitrag Induktion, Exemplifikation und Welterzeugung vorgenommen, eines dieser Grundelemente aufzuspüren und in drei Hauptwerken Goodmans nachzuzeichnen. Ausgangspunkt sind für Ernst Überlegungen Goodmans, die im Zusammenhang mit dem Induktionsproblem angestellt werden. Das Induktionsparadoxon ist berühmt geworden durch die definitorische Einführung des zusammengesetzten Farbprädikats ,grue“ (in der deutschen Übersetzung „,grot“), das zu einem bestimmten Zeitpunkt einen Wechsel der untersuchten Gegenstandsart von grün nach blau prognostiziert - ein Umstand, der von jedem bis zu diesem Zeitpunkt beobachteten grünen Gegenstand der betreffenden Art scheinbar bestätigt wird. Wie diese unsinnige Konsequenz auszuschalten ist, wird für Goodman zur entscheidenden Frage. Ernst zufolge entwickelt Goodman seine Antwort entlang einer signifikanten Gedankenfigur in drei Schritten: 1. Es reicht nicht aus, wahrheitsgemäße Prädikate zu finden, sondern es müssen richtige Kategorien gesucht werden; 2. die richtigen Kategorien werden gefunden, wenn der bisherige Sprachgebrauch betrachtet wird; 3. die Richtigkeit wird vom Sprachgebrauch her bestimmt. Diese Argumentationsstruktur findet Ernst in den Überlegungen zur Exemplifikation in Sprachen der Kunst und zur Unterscheidung richtiger Weltversionen in Weisen der Welterzeugung wieder. Die Richtigkeit von Prädikaten, Kategorisierungen, Systemen wird bestimmt von unserem Gebrauch, von unseren bisherigen Praxen.

Insgesamt wäre es wünschenswert gewesen, wenn weitere Aufsätze den Versuch unternommen hätten, das Verbindende im Werk Goodmans in den Vordergrund zu rücken. Noch immer haben leider zu wenige das Gesamtwerk im Auge, wie Oliver Scholz treffend in der Einleitung bemerkt. Gerade das nach wie vor kaum erschlossene Frühwerk stellt entscheidende Weichen, die das spätere Denken Goodmans maßgeblich beeinflussen; methodische und inhaltliche Gemeinsamkeiten werden erst in der Zusammenschau der auf den ersten Blick stark divergierenden Werke offensichtlich, ebenso wie auch Veränderungen und Verschiebungen erst vor dieser Gegenfolie deutlich hervortreten. Die selektive Rezeption führt leicht zu Missverständnissen und Fehlinterpretationen, und hierin dürfte auch ein Hemmschuh für eine umfassende Rezeption des Werkes Goodmans liegen.

Umso beachtenswerter ist die Leistung des Sammelbandes, der mit den einzelnen Beiträgen alle Werkphasen Goodmans aufgreift und in aktuelle Diskussionszusammenhänge einordnet. Es gibt viel zu entdecken für die Goodman-Interessierten und jene, die sich ein Bild von der Leistungsfähigkeit seiner Theorien machen wollen. Besonders gelobt werden muss, dass es 
den Herausgebern in Zeiten der mangelnden Nachwuchsförderung gelungen ist, neben geschätzten und gut etablierten Wissenschaftlern auch der jüngeren Generation Raum zu geben. So ist nicht nur eine Aufsatzsammlung entstanden, der es gelingt, mit innovativen Fragestellungen aus den Überlegungen Goodmans zu schöpfen, sondern sie zeigt auch einen inspirierenden Querschnitt der Goodmanforschung in Deutschland über Generationen hinweg.

\title{
Eine weder materialistische noch dualistische Theorie des Geistes
}

\author{
Von VITTORIO HÖSLE (Notre Dame)
}

JOHN R. SEARLE: GEIST. Eine Einführung. Suhrkamp Verlag, Frankfurt/M. 2006, 323 S.

Es gehört zu den Wesenszügen eines bedeutenden Philosophen, dass er sich nicht auf eine einzelne Disziplin begrenzt. Denn sosehr die zunehmende Spezialisierung in allen Wissenschaften eine nahe liegende Folge des wachsenden Wissens ist, widerstrebt sie doch dem, was die Philosophie unvermeidlich anstrebt - einem Überblick über das Ganze des Seins. Dass John Searle einer der bedeutendsten lebenden theoretischen Philosophen der USA ist, zeigt sich unter anderem darin, dass er, der schon jung Klassiker der Sprachphilosophie vorgelegt hatte, seit den frühen achtziger Jahren des 20. Jahrhunderts zahlreiche grundlegende Schriften zur Philosophie des Geistes verfasst hat - neben Intentionality (Cambridge 1983) und The Rediscovery of the Mind (Cambridge/Mass. 1992) sowie weiteren Arbeiten, die die Brücke zwischen Sprach- und Geistphilosophie schlagen, nun die hier zu besprechende Einführung.

Diese ist thematisch umfassender als alles Bisherige: „dieses Buch hier ist mein einziger Versuch einer umfassenden Einführung in den gesamten Themenbereich der Philosophie des Geistes." (10) Das Buch ist umso wichtiger, als Searle die Philosophie des Geistes für die wichtigste Disziplin der gegenwärtigen Philosophie hält (16), ja für grundlegender als die Sprachphilosophie (173) - nicht anders als Colin McGinn'; und in der Tat wird man ihm Recht geben, dass die innovativsten und gedankenreichsten Werke der analytischen Philosophie in den letzten Jahrzehnten auf diesem Felde verfasst wurden (vielleicht daneben auch in der Metaphysik und Philosophie der Logik, doch schwerlich noch in der Sprachphilosophie und kaum in Ethik und Politischer Philosophie). Sie haben Standards an Präzision gesetzt, die auch diejenigen nicht unterbieten sollten, die der Ansicht sind, dass die analytische Philosophie bestimmte Denkmöglichkeiten ausklammert, die durchaus wert sind, keineswegs nur in historischer Gelehrsamkeit erinnert, sondern argumentativ wiederbelebt zu werden - ich denke einerseits an eine Philosophie in der Ersten Person wie diejenige Husserls, andererseits an eine Metaphysik des Geistes nach Art Hegels, die gegenüber der transzendentalen Phänomenologie Husserls den enormen Vorteil hat, nicht mit einer Asymmetrie zu Gunsten der eigenen Subjektivität belastet zu sein. Doch hält Searle den Idealismus - er unterscheidet nicht zwischen subjektivem und objektivem Idealismus - für erledigt (56), auch wenn er anerkennt, dass der Dualismus schwerer zu widerlegen ist als der Materialismus, der an der unleugbaren Existenz von Subjektivität scheitert (142). 